\title{
Update on chronic nonspecific lower back pain: rehabilitation
}

\section{AtUALIZAÇÃo EM LOMBALGIA INESPECífICA CRÔNICA: REABILITAÇÃo}

Wanderley M. Bernardo, Roberto Del Valhe Abi Rached, Chennyfer Dobbins Paes da Rosa, Fabio Marcon Alfieri, Silvia Maria

Camillo Amaro, Bruno Nogueira, luciana Dotta, Marta Imamura, linamara Rizzo Battistella, Nathalia Carvalho de Andrada.

1. What is the benefit of acupuncture in the treatment of chronic nonspecific lower back pain?

a. The performance of acupuncture combined with other conservative therapies is not more beneficial than applying the same therapies in isolation

b. Real acupuncture (deep insertion into acupoints) is superior to sham acupuncture (superficial insertion at sites away from the acupoints)

c. Acupuncture is less effective than TENS

d. Acupuncture is less effective than massage

2. What are the advantages of the combination of analgesics and a muscle relaxant?

a. Tizanidine $(2 \mathrm{mg} /$ day $)+$ diclofenac $(50 \mathrm{mg} /$ day $)$ for eight days, is well tolerated by the elderly

b. Carisoprodol has a potential for psychological and physical dependence

c. Analgesic efficacy is lower

d. The number of adverse events is significantly lower

3. Which physical resources are used?
a. The use of shortwave diathermy is recommended
b. Immersion in medicinal water is not recommended
c. The use of continuous ultrasound is not recommen- ded

d. Short waves are less effective than ultrasound

4. What is the role of electrical stimulation in chronic nonspecific lower back pain?
a. TENS can be recommended regardless of physical exercise
b. Electrical stimulation is effective in the absence of exercise-based rehabilitation
c. Massage is more effective than TENS
d. TENS has the advantage to be taken at home

5. Is the use of antidepressants effective for treating non-specific lower back pain?

a. Combined administration of antidepressants with adrenergic effects produces better results than the use of selective serotonin reuptake inhibitors

b. Using for a period longer than three months is recommended

c. Escitalopram at a dose of $20 \mathrm{mg} /$ day is superior to duloxetine

d. Duloxetine at a dose of $5 \mathrm{mg} /$ day is recommended as a first choice

\section{Answers to Clinical Scenario: Update on Primary Dysmenorrhea: Treatment [Published in RAMB 2013; 59(5)]}

1. Is the use of Etoricoxib in the treatment of primary dysmenorrhea more effective than Naproxen? The analgesic efficacy is similar (Alternative B)

2. In women with primary dysmenorrhea, does the use of Valdecoxib have analgesic efficacy superior to Naproxen?

The analgesic efficacy is similar (Alternative A)

3. In women with dysmenorrhea, does the use of Rofecoxib have efficacy superior to Naproxen? The analgesic efficacy is similar (Alternative C)

4. In women with primary dysmenorrhea, does the use of Lumiracoxib have efficacy superior to $\mathrm{Na}$ proxen?

The analgesic efficacy is similar (Alternative D)

5. Is the use of Piroxicam in the treatment of primary dysmenorrhea more effective than Ibuprofen? The analgesic efficacy is similar (Alternative B) 